\title{
Special Issue on Advances in Cardiovascular Biomechanics
}

\author{
J.F. Wenk ${ }^{1} \cdot$ S.M. Lessner ${ }^{2}$ \\ Published online: 4 January 2021 \\ (C) Society for Experimental Mechanics 2021
}

It gives us great pleasure to introduce this Special Issue of Experimental Mechanics focused on Cardiovascular Biomechanics. In this issue, we present a sampling of research in the field where experimental approaches to material characterization play a key role. While the materials and structures of interest may be unfamiliar to those outside our field - fibrous proteins such as collagen and elastin, tissues and organs including the myocardium, heart valves, and blood vessels throughout the body - the processes we investigate are familiar to those of you whose interests lie in classical engineering materials. In particular, processes of material degradation, damage, and failure often lead to severe clinical consequences in biological systems: an example is the rupture of abdominal aortic aneurysms, an issue addressed by Pavey and colleagues in the Wagenseil group in their study of the elastin stabilizing agent pentagalloyl glucose, as well as in a study by Lane and colleagues on the relationship of aneurysm curvature and rupture. Many biological soft tissues are fiber-reinforced composite materials arranged in sheets, and delamination of layers is an important failure mechanism investigated by the Avril group in France in their study of aortic wall dissection. The microstructural aspects of this failure mechanism are further elucidated by the work of Wang and colleagues at Boston University, focusing on the importance of elastin fibers in interlamellar bonding. Other heterogeneities in soft tissues include the interaction of stiff calcifications and fibrous matrix material, which was investigated by Furtunato and colleagues with regard to failure in cerebral tissue.

Of course, living biological tissues also undergo dynamic processes not seen in engineering materials, including growth

J. F. Wenk

jonathan.wenk@uky.edu

1 Department of Mechanical Engineering and Department of Surgery, University of Kentucky, Lexington, KY, USA

2 Department of Cell Biology and Anatomy, Biomedical Engineering Program, University of South Carolina School of Medicine, Columbia, SC, USA and remodeling, a change in material properties and/or geometry resulting from selective addition or removal of specific components. Changes in the mechanical environment can drive tissue growth, as in the study by Dr. Lee's group at Michigan State, and these effects can be important not only in normal physiological conditions such as in response to exercise but also in clinically significant pathologies such as cardiac hypertrophy and heart failure. Along those lines, Grobbel and colleagues investigated the effects of hypertension in remodeling collagen networks and myocytes, and their role in altering the residual stresses in cardiac tissue. The impact of remodeling in the presence of hypertension and pharmacological treatment was studied by Wilson and colleagues, who utilized various imaging techniques to assess changes in extracellular matrix and laminar organization. Tissue remodeling can also include adaptive alterations in geometry, as shown for example in the study by Sang and colleagues of dynamic changes in experimentally-induced rabbit aneurysms, and/or changes in material properties, as noted by Moreno and colleagues in their investigation of arterial stiffening in a mouse model of pulmonary artery hypertension, an increasingly prevalent condition with poor clinical prognosis. Evaluating the effects of remodeling is complicated by intrinsic variations in material properties throughout individual vascular beds, as noted in the study of regional biomechanics and tissue structure of the pulmonary vasculature by Pillalamarri and colleagues.

The techniques used for mechanical characterization of soft tissues range from classical approaches such as the ring-pull assay, here updated and refined in a contribution from the Barocas group, to more recent innovations such as micro computed tomography (microCT). This latter approach has proven to be useful both for accurate geometrical reconstruction of complex structures such as heart valves, as reported in the article by Stephens and colleagues, and for quantitative evaluation of material deformation under pressure loading, as demonstrated in the study of notch propagation in porcine carotid arteries by Brunet and colleagues. Both these studies emphasize the importance of appropriate choice of contrast agents for microCT imaging of soft tissues. The effect of 
calcifications on carotid atherosclerotic plaque was investigated by Cahalane and colleagues by using microCT to identify calcific areas and nanoindentation to characterize the mechanical properties. A unique approach to estimation of local strains in the arterial wall is provided in the work by Rowland, Bailey and Weinberg, who used the relative spacing of endothelial cell nuclei at various pressures to track displacements. Cheuk and colleagues at the Auckland Bioengineering Institute in New Zealand developed a "markerless" technique, using an optical transmission microscope and new digital image correlation algorithms, to measure strain patterns in contracting cardiac muscles. In a separate work to characterize cardiac muscle contraction, Choi and colleagues used electrically stimulated ventricular trabeculae and force measurements to understand the relationship between muscle size and oxygen diffusion. With respect to valvular tissue, Bender and colleagues investigated the effects of radiofrequency ablation on mitral valves by using another advanced imaging technique, multiphoton microscopy, to assess structural changes and biaxial testing to estimate mechanical properties. In a study looking at the relationship between tricuspid valve chordae, Smith and colleagues used uniaxial mechanical testing to assess the stress-strain response and appropriate hyperelastic constitutive models.

Finally, although most of the work presented here involves measurements performed in excised tissues or organs, there is great interest and scope for innovation - as well as unique challenges - in developing methods to measure mechanical properties in living animals or humans. These challenges are evidenced in the contribution by Schreier and colleagues, in which mouse pulmonary artery pressures and blood flow were measured simultaneously in vivo to analyze changes in vascular compliance and wave reflections. Additionally, Aly and colleagues at the University of Pennsylvania used real-time 3D echocardiography to characterize the in vivo dynamics of healthy and ischemic mitral valves. With the ultimate goal of developing non-invasive methods to measure physiologically important mechanical parameters in living human beings, there remains vast potential and significant medical need for novel approaches and technologies.

Publisher's Note Springer Nature remains neutral with regard to jurisdictional claims in published maps and institutional affiliations. 